\title{
La estructura empresarial mexicana de 2003 a 2008: hacia la cuarta década perdida
}

\author{
Gaspar Núñez R.*
}

\begin{abstract}
Resumen
En este artículo analizamos, utilizando la información de los Censos Económicos 2004 y 2009, la estructura empresarial de México y su desempeño en los años 2003 y 2008. El principal hallazgo es que la ya de por sí pobre estructura empresarial existente en 2003, presenta un deterioro aún mayor en 2008. El análisis detallado de las variables relevantes para el desempeño y potencial desarrollo de la economía, confirma que la década 2001-2010 efectivamente puede caracterizarse como "la tercera década perdida", y que la situación actual de la estructura empresarial imposibilita un desarrollo socioeconómico significativo para la década 2011-2020. Los resultados también confirman una de las principales hipótesis de la literatura actual: que el estancamiento económico tiene como una de sus principales causas directas o inmediatas, la insuficiente inversión en capital físico.
\end{abstract}

\begin{abstract}
This research examines Mexican firm structure and performance during the years 2003 and 2008, using data from the 2004 and 2009 Economic Census. We find that the poor firm structure of year 2003 is even worst in 2008. Detailed analysis of variables relevant for economic performance and future development, confirms that the period 2001-2010 was indeed a third lost decade, and that the current firm structure makes it impossible to achieve a meaningful economic development for the 2011-2020 decade. Results also confirm one of the main thesis in current literature, namely, that lack of investment in physical capital is one of the main causes of economy stagnation.
\end{abstract}

Palabras clave: México, estructura empresarial, pyme, desarrollo económico, estancamiento, década perdida.

Key words: Mexico, firm structure, SME, economic development, stagnation, lost decade.

* Miembro del Sistema Nacional de Investigadores de México. Las opiniones expresadas son sólo responsabilidad del autor. 
espués del "milagro mexicano" - ocurrido entre los años cuarenta y los setenta - , México sufrió, junto con casi la totalidad los países latinoamericanos, la "década perdida de los ochenta"; pero a diferencia de otros países, no ha logrado recuperar un crecimiento sólido y consistente.

Como todos los fenómenos complejos, el desarrollo social y económico de un país obedece a diversas causas y responde a múltiples factores; en este artículo postulamos que una condición necesaria es el desarrollo de una estructura empresarial sólida y dinámica.

El análisis que hacemos aquí de los datos proporcionados por los Censos Económicos 2004 y 2009, comprueba que la década 2001-2010 fue, en efecto, otra década perdida, y nos permite argumentar que la década 2011-2020 será también una década perdida en términos de un desarrollo económico sustantivo; sin embargo, la actual puede ser todavía una "década ganada", en la medida en que se comiencen a sentar las bases para emprender un desarrollo sólido y duradero, lo cual depende en gran medida de la evolución política del país.

El artículo se organiza como sigue. En la siguiente sección fundamentamos los términos con que nos referimos a las "tres décadas perdidas". En la segunda sección damos una visión panorámica de la estructura empresarial mexicana, para situar el análisis más detallado de las secciones tercera, cuarta y quinta donde se estudian el sector servicios, el comercio y las manufacturas. En la sección sexta finalizamos con algunas conclusiones y reflexiones.

\section{Las tres décadas perdidas}

Después del milagro mexicano - también denominado periodo de desarrollo estabilizador, de proteccionismo, o de sustitución de importaciones - , la economía mexicana comenzó un periodo de estancamientos y retrocesos que dieron lugar, primero, a la famosa década perdida de los ochenta, y luego a que también se perdieran las dos décadas siguientes.

De acuerdo con Bergoeing et al. (2001: 1), el producto per cápita en México nunca se recuperó, y aún dos décadas después se encontraba un $30 \%$ por debajo de la tendencia anterior. Aunque el criterio utilizado por estos autores es limitado - producto por persona en edad laboral - , constituye uno de los resultados de la literatura que indican la falta de desarrollo 
durante dos décadas en México. Dos estudios más que también indican la carencia de un avance en México durante la década de los noventa, son los de Easterly (2001) y de Santiso (2003).

Como se puede ver en la cita anterior, la referencia a una "década perdida" se hace en términos del crecimiento económico, lo que a su vez se refiere, generalmente, al crecimiento del producto per cápita. En este artículo utilizamos, en su lugar, el concepto de desarrollo económico, en el sentido de mejoramiento continuo de la estructura productiva de la economía - incluido el capital humano - , pues es claro que el producto puede crecer sin que necesariamente se desarrolle la estructura productiva; por otra parte, el desarrollo económico sí implica al crecimiento. En lo que resta de la sección, argumentamos que la década 2001-2010 también fue una década perdida para el desarrollo de México.

En una entrevista reciente, el economista José Luis Calva, con motivo del $5^{\circ}$ informe de gobierno de Felipe Calderón (FC), declaró que de 2007 a 2010 el crecimiento del PIB per cápita fue negativo, que se incrementó la pobreza, y que en materia de empleos, de diciembre de 2006 a julio de 2011, FC generó 751 mil puestos de trabajo permanentes: "Nada, si lo que se requiere es crear al menos 1.3 millones [...] anuales, [en casi cinco años] ni siquiera pudo cumplir con la meta anual" (La Jornada, 4 de noviembre de 2011: 5).

Según otros expertos consultados, la misma nota periodística destaca un crecimiento negativo del PIB per cápita de $-0.48 \%$, un "rotundo fracaso" en la generación de empleos, y una caída real de $3.32 \%$ en el poder adquisitivo de los salarios. También de acuerdo con esa nota, especialistas de la Universidad Nacional Autónoma de México y de la Universidad Autónoma Metropolitana, aseguraron que "son los hechos y las cifras, no los análisis subjetivos, los que claramente reprueban su gestión [de FC], que sin duda pasará a la historia como un sexenio perdido para el desarrollo de México" (idem).

Además de alrededor de 70 mil muertos - causados por la "guerra" calderonista contra el crimen organizado, guerra que incluyó como "daños colaterales" el asesinato de miles de niños y familias enteras no vinculadas a la delincuencia - , lo que aunado a la falta de oportunidades de educación y de trabajo, así como a la falta de un entorno saludable, ha generado una fuerte descomposición social.

Por otra parte, el sexenio anterior (2000-2006), administrado por Vicente Fox (VF), fue otro "sexenio perdido". En efecto, uno de los principales indicadores está dado por un éxodo sin precedentes: alrededor de tres millones y medio de mexicanos - a un ritmo de más de medio millón por añoemigraron en busca de un trabajo que les permitiera subsistir. 
Otro indicador sintomático se encontró en la Encuesta Nacional de Ingreso-Gasto de los Hogares de 2005 (ENIGH-2005, INEGI). El INEGI había venido realizando esta encuesta cada dos años en años pares; pero la titular de la Secretaría de Desarrollo Social - Josefina Vázquez Mota, que fue aspirante a la candidatura por el PAN para la presidencia de la república gestionó la realización de una encuesta extraordinaria en 2005, debido al interés presidencial por mostrar datos positivos con miras a las elecciones presidenciales de 2006. Pero los resultados fueron negativos, luego secuestrados, y sólo se dieron a conocer hasta después de las cuestionadas elecciones, por cuanto indicaban que efectivamente la pobreza y la desigualdad habían aumentado también durante ese año.

En el sexenio de VF se tuvieron los más altos ingresos en la historia de México por concepto de exportación de hidrocarburos: 450 mil millones de dólares, según el entonces gobernador del Banco de México, Guillermo Ortiz. Estos enormes recursos fueron principalmente destinados al despilfarro y a la corrupción para el debilitamiento y manipulación de las instituciones públicas. Tres instituciones fueron las principales afectadas: la Procuraduría General de la República, que se convirtió en una agencia de persecución y acoso de la oposición política; la Suprema Corte de Justicia, que se convirtió en un garante de la impunidad; y las instituciones electorales - Instituto Federal Electoral y Tribunal Federal Electoral - , que se convirtieron en "instituciones" legitimadoras de procesos electorales de dudosa validez.

Siendo la consolidación de instituciones de procuración de justicia y de sistemas electorales de probada credibilidad, elementos esenciales para la prevalencia del Estado de derecho y para la cohesión democrática necesaria en la elección de gobernantes comprometidos con el desarrollo del país, el golpe asestado por VF a las instituciones mexicanas dio lugar a una involución por demás retrógrada de lo que pudo haber sido una prometedora democracia.

Lejos de "los hechos y las cifras" a que aluden los especialistas arriba citados, el discurso oficial, y el presidencial en particular, se empeña en trascender hechos y cifras, pretendiendo dibujar un México que pocos mexicanos creerían. Basten dos ejemplos. Desde la primera mitad del sexenio de VF se acuñó el término "foxilandia", popularmente utilizado para referirse a la tierra o país al que se referían sus discursos, país que poco o nada tenía que ver con la realidad mexicana. El segundo ejemplo tiene que ver con la campaña televisiva impuesta por FC con motivo de su $5^{\circ}$ informe: uno de los principales y grandes "logros", repetido hasta el hartazgo en canales públicos y privados, fue el de tres millones de viviendas construidas 
para los mexicanos durante su gestión, cifra histórica según el presidente. Sin embargo, la realidad mexicana se niega a transformarse a gusto de los decretos presidenciales, y ya desde antes se había anunciado la existencia de cinco millones de casas abandonadas en el país (Estudio Nacional de Vivienda Deshabitada, Comisión de Vivienda de la Cámara de Diputados. Diario La Jornada, 13/08/11: 14.); pero eso no es lo peor, pues "las casas que sale promocionando Calderón en sus comerciales serán derrumbadas, ya que presentan riesgos para sus habitantes" (Héctor García de Ecatepec, La Jornada, 7/09/11: 8).

Por último, citamos un reciente documento - publicado por la Cepal después de la elaboración del presente artículo - en cuya tercera parte, sección "F. Tres décadas de estancamiento económico en México", MorenoBrid y Ros-Bosch (2011) argumentan también que efectivamente México ha sufrido un estancamiento de tres décadas, y que la principal causa directa es la baja tasa de inversión en capital físico, lo cual, como veremos, coincide con el análisis y las conclusiones que elaboramos para un periodo posterior al que ellos consideran.

\section{La estructura empresarial mexicana: panorama nacional}

Según los Censos Económicos 2009, que recogen datos del 2008, en México las pymes constituyen $4.6 \%$ del total de las empresas, y concentran $32.9 \%$ del personal ocupado; es decir: las pymes generan uno de cada tres empleos en México, en donde el 95.2\% de las empresas están en el estrato micro (EMi) y emplean a $45.6 \%$ del personal ocupado. El resto de las empresas $(0.2 \%)$ son empresas grandes que generan el otro $21.5 \%$ del empleo en el país.

La clasificación de las empresas por número de empleados, considerada para los Censos Económicos 2009, se presenta en el cuadro 1; y es la misma para los Censos Económicos 2004, excepto que contempla la inclusión de las ventas anuales para definir el estrato de cada empresa.

Cuadro 1. Estratificación de empresas por número de empleados

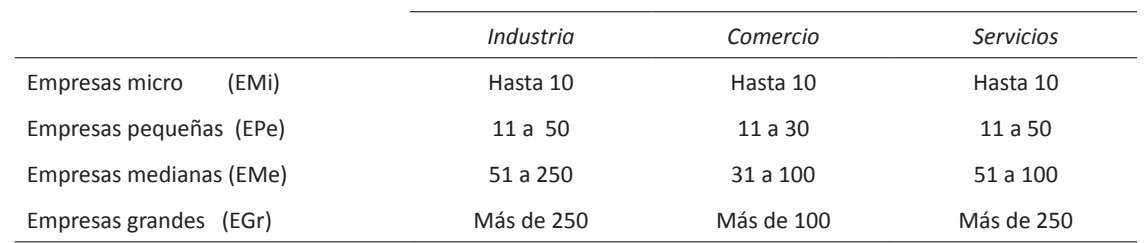

Fuente: Elaborado con base en el Diario Oficial de la Federación, 30/jun/09. 
La información estadística utilizada en nuestro análisis proviene de los Censos Económicos 2004 y 2009, y se refiere a las empresas que realizaron actividades durante 2003 y 2008, y al segmento de las empresas captadas por recorrido total (censo) del sector privado y paraestatal, independientemente de su actividad económica. No se consideran las empresas captadas por muestreo en áreas rurales.

En los Censos Económicos 2004 se encontraron 4290108 unidades económicas en el país. De este universo, para 3495613 empresas, la información se obtuvo mediante censo (recorrido total), y para 794495, mediante muestreo en áreas rurales. De la información captada mediante censo, 3005157 empresas pertenecen al sector privado y paraestatal y operaron en el año 2003; éstas son las que analizamos. El resto de las unidades económicas (490456) pertenecen a servicios del sector público, asociaciones y organizaciones religiosas y unidades que iniciaron sus actividades en 2004 .

En los Censos Económicos 2009 se encontraron 5144056 unidades económicas, de las cuales, 4724892 realizaron actividades en 2008, y 419164 iniciaron actividades en 2009. De las primeras, 3948021 fueron captadas por recorrido total (censo), y 776871 por muestreo. De las captadas por censo, 3724019 pertenecen al sector privado y paraestatal, y son las que aquí analizamos. El resto (224002 empresas) pertenecen al sector público y a asociaciones religiosas (INEGI, 2010a).

A lo largo del estudio, además de excluir al gobierno, a las asociaciones religiosas y a las organizaciones extraterritoriales, como ya se dijo, los totales nacionales excluyen también al sector agropecuario, de por sí excluido de los censos económicos.

El cuadro 2 muestra la estructura nacional que las unidades económicas tenían en 2003 y 2008, según estrato por número de empleados (personal ocupado), así como el incremento de empresas en ese periodo. Aunque en términos absolutos se observan cambios - el número de empresas aumenta en 718862 - , las participaciones porcentuales de cada estrato indican que la estructura empresarial se mantuvo prácticamente invariable a nivel nacional, y salta a la vista el hecho de que las EMi constituyen 95\% del total; es decir, las pymes (pequeñas y medianas) y las grandes empresas, sólo tienen $5 \%$ de todas las empresas del país.

De las 718862 empresas creadas, 682887 fueron EMi; o sea: sólo 5\% (35975) fueron pymes o grandes; esto tiene consecuencias fundamentales para la economía y su potencial desarrollo, pues como veremos, en México el grueso de las EMi son empresas pobres en varios sentidos, y lejos de constituir potenciales soportes para el desarrollo del país, son más bien válvulas de escape a las condiciones nacionales de desempleo y pobreza. 
Cuadro 2. Empresas a nivel nacional, por estrato empresarial

\begin{tabular}{lrrrrrr}
\hline & \multicolumn{2}{c}{ Año 2003} & & \multicolumn{2}{c}{ Año 2008 } & Crecimiento \\
\cline { 2 - 3 } & \multicolumn{1}{c}{ Total } & $\%$ & & Total & $\%$ & 2003-2008 (\%) \\
\hline Nacional & 3005157 & 100.0 & & 3724019 & 100.0 & 23.90 \\
Micro & 2853291 & 94.9 & & 3536178 & 95.0 & 23.90 \\
Pequeña & 118085 & 3.9 & & 149968 & 4.0 & 27.00 \\
Mediana & 27073 & 0.9 & & 30697 & 0.8 & 13.40 \\
Grande & 6708 & 0.2 & 7176 & 0.2 & 0.70 \\
\hline
\end{tabular}

Fuente: Elaborado con datos de los Censos Económicos 2004 y 2009 del INEGI.

El hecho de que incrementen su participación, mientras que el resto disminuye o se estanca, implica entonces un deterioro de la estructura empresarial necesaria para el desarrollo económico, pues el robusto desarrollo de una economía no puede sustentarse sobre un sustrato empresarial cada vez más empobrecido.

Por otra parte, el incremento en la participación de las pymes, que constituyen una columna vertebral del desarrollo de las economías de mercado, muestra en México un aumento mínimo en las pequeñas empresas y, peor aún, una disminución de 0.9 a 0.8 en el caso de las medianas.

El cuadro 3 tiene la composición del empleo a nivel nacional. Como puede observarse, a pesar de los cambios, la estructura es esencialmente la misma en 2003 y en 2008. Las EMi tienen más empleados, seguidas por las grandes empresas, y luego por las pymes: las EMi elevan su participación en el total de $38.3 \%$ en 2003 , a $41.8 \%$ en 2008; es decir, se observa un incremento de la participación de las EMi en el empleo, y un decremento similar en las grandes y medianas empresas; las pequeñas mantienen la misma participación. Esto significa que el empleo en las EMi crece más que proporcionalmente, lo que implica una pauperización creciente de los empleos, con las consiguientes implicaciones negativas.

Cuadro 3. Empleados por estrato a nivel nacional

\begin{tabular}{lcccccc}
\hline & \multicolumn{2}{c}{ Año 2003 } & & \multicolumn{2}{c}{ Año 2008 } & Crecimiento \\
\cline { 2 - 3 } & Personal ocupado & $\%$ & & Personal ocupado & $\%$ & 2003-2008 (\%) \\
\hline Total & 16239536 & 100.0 & & 20116834 & 100.0 & 19.3 \\
Micro & 6224965 & 38.3 & & 8414444 & 41.8 & 26.0 \\
Pequeña & 2478964 & 15.3 & & 3078665 & 15.3 & 19.5 \\
Mediana & 2828306 & 17.4 & & 3199650 & 15.9 & 11.6 \\
Grande & 4707301 & 29.0 & & 5424075 & 27.0 & 13.2 \\
\hline
\end{tabular}

Fuente: Elaborado con datos de los Censos Económicos 2004 y 2009 del INEGI. 
Los tres grandes sectores de actividad económica más importantes del país son la industria manufacturera, el comercio, y el sector servicios, que en su conjunto poseían, en 2003, 97.2\% de las unidades económicas del país, y $88.8 \%$ del personal ocupado (INEGI, 2006: 13).

El cuadro 4 presenta a las empresas por sector de actividad. El comercio agrupa la mayor cantidad de empresas; le siguen el sector servicios y las manufacturas. Para 2008, comercio, servicios y manufacturas concentraban $98.3 \%$ del total de las empresas. Notemos que los servicios presentan el mayor incremento en su participación.

El cuadro 5 presenta el personal ocupado por actividad económica. Los servicios tienen la mayor cantidad de empleados, y le siguen el comercio y las manufacturas. Para 2008, comercio, servicios y manufacturas concentraban $90.2 \%$ del personal ocupado.

En cuanto al crecimiento, el personal ocupado aumentó en $23.9 \%$, observándose el mayor crecimiento en los servicios, lo cual podría interpretarse como una "terciarización" de la economía; pero tal crecimiento se basa en el aumento de EMi, lo que en México significa que se da una "changarrización" de la economía, con los consecuentes efectos negativos. ${ }^{1}$

\section{Cuadro 4. Unidades económicas por sector de actividad económica}

\begin{tabular}{|c|c|c|c|c|c|}
\hline & \multicolumn{2}{|c|}{ Año 2003} & \multicolumn{2}{|c|}{ Año 2008} & \multirow{2}{*}{$\begin{array}{c}\text { Crecimiento } \\
2003-2008(\%)\end{array}$} \\
\hline & Empresas & $\%$ & Empresas & $\%$ & \\
\hline Total nacional & 3005157 & 100.0 & 3724019 & 100.0 & 23.9 \\
\hline Servicios & 1013743 & 33.7 & 1367287 & 36.7 & 34.9 \\
\hline Comercio & 1580587 & 52.6 & 1858550 & 49.9 & 17.6 \\
\hline Industria manufacturera & 328718 & 10.9 & 436851 & 11.7 & 32.9 \\
\hline Construcción & 13444 & 0.4 & 18637 & 0.5 & 38.6 \\
\hline Transp. correos y almacen. & 41899 & 1.4 & 17705 & 0.5 & -57.7 \\
\hline Electricidad agua y gas & 2437 & 0.1 & 2589 & 0.1 & 6.2 \\
\hline Pesca y acuicultura animal & 21252 & 0.7 & 19443 & 0.5 & -8.5 \\
\hline Minería & 3077 & 0.1 & 2957 & 0.1 & -3.9 \\
\hline
\end{tabular}

\%: Participación relativa de cada sector en el total nacional.

Fuente: Elaborado con datos de los Censos Económicos 2004 y 2009.

1 "Changarrización" es un término que surgió a partir de la pretensión de la administración de Vicente Fox de resolver el desarrollo socioeconómico del país con microcréditos que permitieran a los emprendedores mexicanos establecer sus "changarros", término popular - despectivo en cierto grado - usado para referirse a negocios pequeños y precarios. 
Cuadro 5. Personal ocupado por sector de actividad económica

\begin{tabular}{|c|c|c|c|c|c|}
\hline & \multicolumn{2}{|c|}{ Año 2003} & \multicolumn{2}{|c|}{ Año 2008} & \multirow{2}{*}{$\begin{array}{c}\text { Crecimiento } \\
2003-2008 \\
(\%)\end{array}$} \\
\hline & Empleados & $\%$ & Empleados & $\%$ & \\
\hline Total nacional & 16239536 & 100.0 & 20116834 & 100.0 & 23.9 \\
\hline Servicios & 5215808 & 32.1 & 7340216 & 36.5 & 40.7 \\
\hline Comercio & 4997366 & 30.8 & 6134758 & 30.5 & 22.8 \\
\hline Industria manufacturera & 4198579 & 25.9 & 4661062 & 23.2 & 11.0 \\
\hline Construcción & 652387 & 4.0 & 704640 & 3.5 & 8.0 \\
\hline $\begin{array}{l}\text { Transporte, correos y } \\
\text { almacenamiento }\end{array}$ & 634940 & 3.9 & 718062 & 3.6 & 13.1 \\
\hline Electricidad, agua y gas & 221335 & 1.4 & 235688 & 1.2 & 6.5 \\
\hline $\begin{array}{l}\text { Pesca y acuicultura } \\
\text { animal }\end{array}$ & 196481 & 1.2 & 180083 & 0.9 & -8.3 \\
\hline Minería & 122648 & 0.8 & 142325 & 0.7 & 16.0 \\
\hline
\end{tabular}

Fuente: Elaborado con datos de los Censos Económicos 2004-2009.

En suma, manufacturas, comercio y servicios concentraban, en 2008, 98.3\% de las empresas y $90.2 \%$ del personal ocupado, por lo cual analizamos la economía a partir de esos tres grandes sectores. En lo que resta de la sección, definimos el universo constituido por los tres sectores.

El cuadro 6 presenta la composición de la participación en el total de unidades económicas, por tamaño de empresa en los tres sectores. De 2923048 unidades económicas en 2003, 95.5\% eran micro empresas, 3.5\% pequeñas empresas, y $0.8 \%$ y $0.2 \%$ eran, respectivamente, empresas medianas y grandes. Para 2008, el número de unidades crece en 25.3\% (739297 empresas más), pero la composición es básicamente la misma, y el grueso del aumento se da en las microempresas: había 706357 EMi más que en 2003.

El cuadro 7 presenta la composición del personal ocupado total, por estrato empresarial en los tres sectores. De 14411753 empleados en 2003,

Cuadro 6. Empresas en la manufactura, comercio y servicios

\begin{tabular}{|c|c|c|c|c|c|}
\hline & \multicolumn{2}{|c|}{ Año 2003} & \multicolumn{2}{|c|}{ Año 2008} & \multirow{2}{*}{$\begin{array}{c}\text { Crecimiento } \\
\text { 2003-2008 (\%) }\end{array}$} \\
\hline & Empresas & $\%$ & Empresas & $\%$ & \\
\hline Total & 2923048 & 100.0 & 3662345 & 100.0 & 25.3 \\
\hline Micro & 2792678 & 95.5 & 3499035 & 95.5 & 25.3 \\
\hline Pequeña & 102589 & 3.5 & 132127 & 3.6 & 28.8 \\
\hline Mediana & 22081 & 0.8 & 25454 & 0.7 & 15.3 \\
\hline Grande & 5700 & 0.2 & 5729 & 0.2 & 0.5 \\
\hline
\end{tabular}

Fuente: Elaborado con datos de los Censos Económicos 2004-2009. 


\begin{tabular}{|c|c|c|c|c|c|c|}
\hline \multirow{4}{*}{ 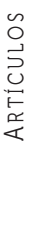 } & \multicolumn{6}{|c|}{ Cuadro 7. Personal ocupado en la y manufactura, comercio servicios } \\
\hline & & \multicolumn{2}{|c|}{ Año 2003} & \multicolumn{2}{|c|}{ Año 2008} & \multirow{2}{*}{$\begin{array}{c}\text { Crecimiento } \\
2003-20081 \%\end{array}$} \\
\hline & & Empleados & $\%$ & Empleados & $\%$ & \\
\hline & Total & 14411753 & 100.0 & 18285290 & 100.0 & 25.8 \\
\hline & Micro & 6061731 & 42.1 & 8285290 & 45.7 & 36.7 \\
\hline \multirow{3}{*}{ 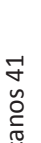 } & Pequeña & 2118661 & 14.7 & 2663693 & 14.7 & 25.7 \\
\hline & Mediana & 2326881 & 16.1 & 2673718 & 14.7 & 14.9 \\
\hline & Grande & 3904480 & 27.1 & 4513335 & 24.9 & 15.6 \\
\hline
\end{tabular}

Fuente: Elaborado con datos de los Censos Económicos 2004-2009.

$42.1 \%$ estaba en una EMi; $14.7 \%$ en una pequeña, y 16.1 y $27.1 \%$ en una mediana y gran empresa, respectivamente.

Para el 2008, la composición estructural es básicamente la misma, pero el estrato micro incrementa su participación en la medida en que las pymes y las grandes empresas la disminuyen, en consonancia con la "changarrización" de la economía a que antes nos referimos: las EMi aumentan su participación en 3.6\% (a 47.7\%), a costa de una disminución de 1.4\% y 2.2\% en las medianas y grandes, respectivamente. El crecimiento medio observado en el empleo durante el periodo, $25.8 \%$, se explica lógicamente, en su mayor parte, por el crecimiento de las EMi.

En las siguientes secciones verificaremos que las EMi son un estrato muy empobrecido en varios aspectos, particularmente en lo que se refiere a remuneraciones, producción e inversión; y que con respecto a 2003, en 2008 la estructura empresarial productiva sufrió un retroceso que conduce al menos a dos conclusiones: a) la situación de la estructura empresarial y sus cambios de 2003 a 2008 corroboran que 2001-2010 fue una década perdida, y b) tal estructura implica que, en lo que al desarrollo económico concierne, la década de 2010 también será una década perdida.

\section{La industria manufacturera}

De acuerdo con los datos del cuadro 8, para 2008 la industria tenía $11.7 \%$ de las unidades económicas del país, pero participaba con $44.3 \%$ de la producción total. Por otra parte, empleaba a $23.2 \%$ de todo el personal ocupado, con un promedio de 10.7 empleados por establecimiento. Para la producción total, se observa una participación de $43.3 \%$, con un crecimiento real de $31 \%$.

Los cambios de 2003 a 2008 no son notables. Destaca la disminución en la participación del personal ocupado, que baja en $2.7 \%$, lo cual, como 
Cuadro 8. Manufactura: unidades económicas, personal ocupado y producción

\begin{tabular}{|c|c|c|c|c|c|c|}
\hline & \multicolumn{3}{|c|}{ Año 2003} & \multicolumn{3}{|c|}{ AÑO 2008} \\
\hline & Total nacional & Manufactura & $\%$ & Total nacional & Manufactura & $\%$ \\
\hline Empresas & 3005157 & 328718 & 10.9 & 3724019 & 436851 & 11.7 \\
\hline Empleados & 16239536 & 4198579 & 25.9 & 20116734 & 4661062 & 23.2 \\
\hline Producción* & 6317179 & 2732718 & 43.3 & 8077575 & 3581815 & 44.3 \\
\hline
\end{tabular}

* En millones de pesos constantes de 2003. Pesos de 2008 deflactados con el Índice nacional de precios productor, que se incrementó de 100 en diciembre de 2003 a 136.16 en diciembre de 2008.

$<$ http://dgcnesyp.inegi.org.mx/cgi-win/bdieintsi.exe/Consultar>.

Fuente: Elaborado con datos de los Censos Económicos 2004 y 2009.

veremos en la sección 6, se corresponde con un incremento de la participación del sector servicios de $4.4 \%$. Como dijimos, esto podría interpretarse como una "terciarización" de la economía, pero servicios es un sector empobrecido, lo que lleva a concluir que absorbe mano de obra liberada por una industria manufacturera con problemas para crecer.

El cuadro 9 muestra la estructura empresarial de la industria. El número de empresas aumentó en 108133 (32.9\%), de las que 105478 eran EMi; dicho de otro modo, $97.5 \%$ de las nuevas empresas observadas en 2008 eran EMi, un crecimiento de $35 \%$ que incrementó su participación de $90.9 \%$ en 2003 a $92.5 \%$ en 2008, en el total de las empresas de la industria.

La preponderancia de las EMi es abrumadora; presentaron un crecimiento relativo de $13.1 \%$ (2595 unidades) y, en comparación, el cambio en el número de medianas y grandes no es significativo, y este estancamiento constituye un retroceso. El crecimiento de las EMi impresiona, porque implica un fuerte impacto negativo sobre la estructura empresarial de la industria; como veremos al analizar las remuneraciones y la formación

Cuadro 9. Manufacturas: Empresas por estrato

\begin{tabular}{lccccc} 
& \multicolumn{2}{c}{ Año 2003 } & \multicolumn{2}{c}{ Año 2008 } & Crecimiento \\
\cline { 2 - 4 } & Empresas & $\%$ & Empresas & $\%$ & 2003-2008 (\%) \\
\hline Manufactura & 328718 & 100.0 & 436851 & 100.0 & 32.9 \\
Micro & 298678 & 90.9 & 404156 & 92.5 & 35.3 \\
Pequeña & 19754 & 6.0 & 22349 & 5.1 & 13.1 \\
Mediana & 7235 & 2.2 & 7113 & 1.6 & -0.17 \\
Grande & 3051 & 0.9 & 3233 & 0.7 & 6.0 \\
\hline
\end{tabular}

\%: Participación relativa de cada estrato empresarial en el total del sector. Fuente: Elaborado con datos de los Censos Económicos 2004 y 2009. 
bruta de capital fijo (FBCF), esto significa empobrecimiento de empresas y trabajadores.

En el cuadro 10 tenemos los datos sobre empleo: 462483 empleos creados en cinco años, es decir, un promedio anual de 92497, cifra de poco valor dado el tamaño de la economía mexicana y los empleos requeridos. Pero además, 318610 - 68.9\% de esos nuevos empleos - fueron creados por las EMi nuevamente, lo que implica un retroceso.

Cuadro 10. Industria Manufacturera: Personal ocupado

\begin{tabular}{lcccccc}
\cline { 2 - 3 } & \multicolumn{2}{c}{ Año 2003 } & & \multicolumn{2}{c}{ Año 2008 } & Crecimiento \\
\cline { 2 - 3 } Mersonal ocupado & $\%$ & & Personal ocupado & $\%$ & 2003-2008 (\%) \\
\hline Manufactura & 4198579 & 100.0 & & 4661062 & 100.0 & 11.0 \\
Micro & 762103 & 18.2 & & 1080713 & 23.2 & 41.8 \\
Pequeña & 431768 & 10.3 & & 467197 & 10.0 & 8.2 \\
Mediana & 810095 & 19.3 & & 797907 & 17.1 & -1.5 \\
Grande & 2194613 & 52.3 & & 2315245 & 49.7 & 5.5 \\
\hline
\end{tabular}

\%: Participación relativa de cada estrato empresarial en el total del sector.

Fuente: Elaborado con datos de los Censos Económicos 2004 y 2009.

El cuadro 11 presenta el número promedio de empleados por estrato. Para la industria en su conjunto, la media disminuyó de 12.8 empleados por empresa en 2003 a 10.7 en 2008. El punto de interés aquí es que las EMi, estrato que presenta los cambios más importantes de 2003 a 2008, mantiene una media de 2.6 a 2.7 empleados, cuando el rango del estrato va de uno hasta 10 empleados. Aunando esto a la disminución de la media en las grandes y pequeñas empresas, obtenemos otro indicador de la "changarrización" del sector manufacturero.

A partir del cuadro 12, comenzamos a ver los datos que demuestran el paupérrimo estado de las EMi. En este cuadro tenemos los datos sobre

Cuadro 11. Manufacturas: Empleados promedio por empresa

\begin{tabular}{cccc}
\cline { 2 - 4 } & Año 2003 & Año 2008 & Crecimiento 2003-2008 (\%) \\
\hline Manufactura & 12.8 & 10.7 & -16.5 \\
Micro & 2.6 & 2.7 & 4.8 \\
Pequeña & 21.9 & 20.9 & -4.4 \\
Mediana & 112.0 & 112.2 & 0.2 \\
Grande & 719.3 & 716.1 & -0.4 \\
\hline
\end{tabular}

Fuente: Elaborado con datos de los Censos Económicos 2004 y 2009. 
Cuadro 12. Manufactura: personal remunerado

\begin{tabular}{lccccc}
\cline { 2 - 3 } & \multicolumn{2}{c}{ Año 2003 } & & \multicolumn{2}{c}{ Año 2008 } \\
\cline { 2 - 3 } \cline { 5 - 6 } Manufactura & PODRS remun & \%TD & & PODRS remun & \%TD \\
\hline Micro & 3387333 & 87.8 & & 3276685 & 82.1 \\
Pequeña & 307903 & 41.0 & & 387420 & 36.6 \\
Mediana & 386654 & 94.3 & & 395978 & 90.6 \\
Grande & 727194 & 99.4 & & 661578 & 99.5 \\
\hline
\end{tabular}

PODRS remun: Personal ocupado dependiente de la razón social remunerado.

\%TD: Porcentaje del personal ocupado dependiente total.

Fuente: Elaborado con datos de los Censos Económicos 2005 y 2009.

el personal ocupado dependiente de la razón social remunerado y, como es de esperar, el porcentaje del personal remunerado aumenta a medida que el tamaño de las empresas es mayor; dicho de otro modo: las EMi generan menos empleos remunerados, lo que apoya la hipótesis de que son mayormente iniciativas de autoempleo.

La manufactura en su conjunto disminuyó el personal remunerado de $87.8 \%$ en 2003 , a $82.1 \%$ en 2008 , tendencia que muestra el proceso de empobrecimiento estructural de la economía, pues cuanto menor es el porcentaje de personal remunerado, tanto más tenemos EMi con actividad informal. En efecto, si consideramos que el porcentaje para la mediana y la gran empresa se mantiene invariable, mientras que la pequeña $y$, mayormente, la EMi son las que muestran una disminución significativa del empleo remunerado, y que además las EMi presentan el mayor crecimiento en unidades y empleos generados, se deduce con claridad que la estructura de la industria se empobrece.

La diferencia presentada por las EMi es abismal: en 2003 tenían 41\% de personal remunerado, mientras que en las pequeñas empresas observamos un $94.3 \%$. En 2008, estos porcentajes muestran un empeoramiento, pues son, respectivamente, de $36.6 \%$ y $90.6 \%$; es decir: se acerca a tres veces menos personal remunerado; si además consideramos que el personal ocupado promedio en las EMi era de 2.7 empleados, esto significa que, en promedio, cada EMi tiene menos de un empleado remunerado.

En el cuadro 13 tenemos el valor agregado bruto (VAB). Tanto en términos absolutos como en relativos (por empleado), la producción de VAB decrece considerablemente para las EMi y las pequeñas empresas, mientras que para las medianas y grandes presenta un crecimiento moderado; el retroceso de las EMi es dramático: casi $40 \%$ menos VAB por empleado.

El cuadro 14 presenta la Formación Bruta de Capital Fijo (FBCF). La FBCF media en la industria es de 16.4 mil pesos por empleado en 2003, y dismi- 


\begin{tabular}{|c|c|c|c|c|c|c|c|}
\hline \multirow{3}{*}{ 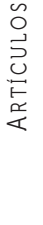 } & \multicolumn{7}{|c|}{$\begin{array}{l}\text { Cuadro 13. Sector Manufacturero: valor agregado bruto (VAB) por estrato } \\
\text { empresarial y por empleado, en pesos constantes de } 2003\end{array}$} \\
\hline & & \multicolumn{2}{|c|}{$\begin{array}{l}\text { VAB por estrato } \\
\text { (Millones de pesos) }\end{array}$} & \multirow{2}{*}{$\begin{array}{c}\text { Crecimiento } \\
2003-2008 \text { (\%) }\end{array}$} & \multicolumn{2}{|c|}{$\begin{array}{l}\text { VAB por empleado } \\
\text { (Miles de pesos) }\end{array}$} & \multirow{2}{*}{$\begin{array}{c}\text { Crecimiento } \\
2003-2008 \text { (\%) }\end{array}$} \\
\hline & & 2003 & 2008 & & 2003 & 2008 & \\
\hline & Manufactura & 927987 & 1087560 & 17.2 & 221 & 233 & 5.6 \\
\hline \multirow{4}{*}{ 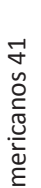 } & Micro & 36150 & 30958 & -14.4 & 47 & 29 & -39.6 \\
\hline & Pequeña & 50797 & 46688 & -8.1 & 118 & 100 & -15.1 \\
\hline & Mediana & 154866 & 190450 & 23.0 & 191 & 239 & 24.9 \\
\hline & Grande & 686175 & 819464 & 19.4 & 313 & 354 & 13.2 \\
\hline
\end{tabular}

Nota: Pesos de 2008 deflactados con el Índice nacional de precios productor, que se incrementó de 100 en diciembre de 2003, a 136.16 en diciembre de 2008.

<http://dgcnesyp.inegi.org.mx/cgi-win/bdieintsi.exe/Consultar>

Fuente: Elaborado con datos de los Censos Económicos 2005 y 2009.

nuye a 14.2 en 2008; es decir, para 2008 el sector manufacturero invertía en FBCF por empleado, 2.1 mil pesos menos que cinco años antes.

En 2003, la FBCF por empleado en las EMi fue de 2.8 mil pesos, y para 2008 disminuye a 1.1 mil pesos, una disminución real de $60.7 \%$. Dado que el número medio de empleados en la EMi fue de 2.7 en 2008, estamos hablando de una inversión media por EMi de 2.9 mil pesos anuales en 2008. Es evidente que una economía con estas tendencias no se está desarrollando; por el contrario, se empobrece estructuralmente. Es bien sabido que una de las claves del desarrollo está en el incremento de la relación capital-trabajo, por lo que la "changarrización" de la estructura empresarial, y el empobrecimiento extremo de la microindustria, claramente constituyen un impedimento y, más aún, un retroceso en el desarrollo de la economía mexicana.

En 2003, la FBCF en la pequeña industria fue 3.6 veces más alta que en las EMi, y aunque en la pequeña industria también disminuye dramática-

Cuadro 14. Sector manufacturero: FBCF por empleado (miles de pesos constantes de 2003)

\begin{tabular}{lccccc}
\hline & \multicolumn{3}{c}{$F B C F$} & & \multicolumn{2}{c}{ Crecimiento } \\
\cline { 2 - 3 } \cline { 5 - 6 } \cline { 5 - 6 } Manufactura & 2003 & 2008 & & Absoluto & $\%$ \\
Micro & 16.4 & 14.2 & & -2.1 & -12.9 \\
Pequeña & 2.8 & 1.1 & & -1.7 & -60.7 \\
Mediana & 10.0 & 4.7 & & -5.3 & -53.1 \\
Grande & 13.9 & 15.9 & & 2.0 & 14.3 \\
\hline
\end{tabular}

Fuente: Elaborado con datos de los Censos Económicos 2004 y 2009. 
mente $(-53.1 \%)$, para 2008 esa relación asciende a 4.3. Esta relación es importante, porque muestra cómo, al pasar de una escala de 2.7 empleados promedio para las EMi, a 20.9 en la pequeña empresa, la FBCF por empleado se cuadruplica por lo menos; tal importancia se deriva del hecho de que el crecimiento de la razón capital-trabajo es un factor fundamental para el desarrollo de una economía, especialmente para el desarrollo de una economía que presenta tan fuertes atrasos en la relación capital-trabajo como la mexicana.

La mediana empresa presenta un moderado crecimiento de $14.3 \%$, invirtiendo en 2008 dos mil pesos más por empleado que en 2003. Aquí es aún más impresionante la diferencia en la FBCF por empleado con respecto a la EMi, al pasar a una escala de 112.2 empleados por unidad económica: las medianas empresas invirtieron por empleado 20 veces más que las microempresas. Esto demuestra la importancia fundamental de desarrollar las pymes, si se quiere impulsar el crecimiento económico.

Veamos ahora las remuneraciones pagadas. Según el cuadro 15, la industria en su conjunto tuvo un decrecimiento de $7.7 \%$ en términos absolutos, explicado por la disminución, en términos reales, de las remuneraciones pagadas por las pymes y las industrias grandes. Por su parte, las EMi presentan un crecimiento positivo, en concordancia con lo antes visto: hay más empresas y más personas, tanto en términos absolutos como relativos, en el estrato micro.

Las últimas tres columnas, relativas a las remuneraciones mensuales por empleado, dan una visión más clara de lo dicho: una fuerte y generalizada disminución de las remuneraciones reales pagadas por la industria manufacturera: de 5811 pesos mensuales en 2003, a 4829 en 2008, lo que significa un decrecimiento de $16.9 \%$. Es notable también que la más impor-

Cuadro 15. Industria manufacturera: remuneraciones por estrato empresarial y por empleado, a pesos constantes de 2003

\begin{tabular}{|c|c|c|c|c|c|c|}
\hline & \multicolumn{2}{|c|}{$\begin{array}{l}\text { Remuneraciones por estrato } \\
\text { (miles de pesos) }\end{array}$} & \multirow{2}{*}{$\begin{array}{l}\text { Crecimiento } \\
2003-2008 \\
(\%)\end{array}$} & \multicolumn{2}{|c|}{$\begin{array}{c}\text { Remuneraciones por } \\
\text { empleado } \\
\text { (pesos mensuales) }\end{array}$} & \multirow{2}{*}{$\begin{array}{c}\text { Crecimiento } \\
2003-2008 \\
(\%)\end{array}$} \\
\hline & 2003 & 2008 & & 2003 & 2008 & \\
\hline Manufactura & 292768582 & 270118132 & -7.7 & 5811 & 4829 & -16.9 \\
\hline Micro & 11509245 & 11601428 & 0.8 & 1258 & 895 & -28.9 \\
\hline Pequeña & 20696001 & 17773805 & -14.1 & 3994 & 3170 & -20.6 \\
\hline Mediana & 56318419 & 48036707 & -14.7 & 5793 & 5017 & -13.4 \\
\hline Grande & 204244917 & 192706191 & -5.6 & 7756 & 6936 & -10.6 \\
\hline
\end{tabular}

Nota: Pesos de 2008 deflactados con el Índice nacional de precios productor, que ascendió de 100 en diciembre de 2003, a 136.16 en diciembre de 2008. http://dgcnesyp.inegi.org.mx/cgi-win/bdieintsi.exe/Consultar Fuente: Elaborado con datos de los Censos Económicos 2005 y 2009. 
tante disminución en las remuneraciones mensuales reales por empleado, se observan en la EMi; es decir, en 2008 había más EMi precarias y eran aún más precarias que antes.

\section{Sector servicios}

En términos del número de unidades económicas y del empleo generado, los servicios representaron más de un tercio de la economía mexicana: este sector empleó a 32.1\% del personal ocupado en 2003, y a 36.5\% en 2008; el número de establecimientos también aumentó su participación nacional al pasar del 33.7\% al 36.7\%. Al mismo tiempo, la participación relativa en el producto bruto disminuyó de $23.3 \%$ a $21.7 \%$, lo que se explica por el incremento de EMi de bajo valor agregado.

En 2008, en el Sector Servicios había tres veces más empresas que en el Sector Manufacturero, pero el promedio de empleados por empresa era de casi la mitad: 10.7 en las manufacturas, y 5.4 en servicios; estas cifras son un indicador de que los servicios, a diferencia de las manufacturas y el comercio, requieren menos capital y, por lo tanto, más personas se establecen como micro-empresarios principalmente en la prestación de servicios menores (cuadro 16).

Cuadro 16. Sector servicios: unidades económicas, personal ocupado y producción

\begin{tabular}{lrrrrrr} 
& \multicolumn{3}{c}{ Año 2003 } & \multicolumn{3}{c}{ Año 2008 } \\
\cline { 2 - 7 } & \multicolumn{1}{c}{ Total país } & Sector Servicios & $\%$ & Total país & Sector Servicios & $\%$ \\
\hline Unidades económicas & 3005157 & 1013743 & 33.7 & 3724019 & 1367287 & 36.7 \\
Personal ocupado & 16239536 & 5215808 & 32.1 & 20116834 & 7340216 & 36.5 \\
Producción bruta* & 63171788 & 1473364.2 & 23.3 & 8077575 & 1749927 & 21.7 \\
\hline
\end{tabular}

\%: Participación relativa del sector Servicios en el total del país.

*En millones de pesos constantes de 2003. Pesos de 2008 deflactados con el Índice nacional de precios productor, que se incrementó de 100 en diciembre de 2003, a 136.16 en diciembre de 2008.

$<$ http://dgcnesyp.inegi.org.mx/cgi-win/bdieintsi.exe/Consultar>

Fuente: Elaborado con datos de los Censos Económicos 2004 y 2009.

La estructura empresarial en los servicios no tuvo variaciones significativas; en todos los casos, los cambios observados en la participación de cada estrato son menores a $1 \%$, como se observa en el cuadro 17. La característica más importante de esta estructura, es que las EMi mantuvieron una participación superior a 94\%, lo cual, en términos del desarrollo eco- 
Cuadro 17. Sector Servicios: unidades económicas por estrato empresarial

\begin{tabular}{lcrrrrrr}
\hline & \multicolumn{2}{c}{ Año 2003 } & & \multicolumn{2}{c}{ Año 2008 } & Crecimiento \\
\cline { 2 - 3 } Servicios & Núm. de empresas & \multicolumn{1}{c}{$\%$} & & Núm. de empresas & $\%$ & 2003-2008 (\%) \\
Micro & 1013720 & 100.0 & & 1367277 & 100.0 & 34.9 \\
Pequeña & 960135 & 94.7 & & 1291080 & 94.4 & 34.5 \\
Mediana & 43835 & 4.3 & 64307 & 4.7 & 46.7 \\
Grande & 5179 & 0.5 & 6555 & 0.5 & 26.6 \\
\hline
\end{tabular}

\%: Participación relativa de cada estrato empresarial en el total sectorial.

Fuente: Elaborado con datos de los Censos Económicos 2004 y 2009.

nómico del país, constituye un indicador negativo, pues como anotamos, cuanto mayor y más persistente es el número de EMi, menor integración y desarrollo se observa en las empresas pequeñas y medianas. El total de empresas del sector se incrementó en 353557, de las que 330945 (93.6\%) eran EMi; esto es: en cinco años sólo se crearon 22612 pequeñas, medianas y grandes empresas.

En el cuadro 18 nuevamente observamos que las EMi ocuparon a la mayor parte de los empleados; esto indica un proceso de pauperización de la economía, en el sentido de que la mayor participación y el mayor crecimiento se dan en el estrato de menor potencial, el de las EMi.

Cuadro 18. Sector servicios: personal ocupado

\begin{tabular}{|c|c|c|c|c|c|}
\hline & \multicolumn{2}{|c|}{ Año 2003} & \multicolumn{2}{|c|}{ Año 2008} & \multirow{2}{*}{$\begin{array}{c}\text { Crecimiento } \\
2003-2008(\%)\end{array}$} \\
\hline & Personal ocupado & $\%$ & Personal ocupado & $\%$ & \\
\hline Servicios & 5215808 & 100.00 & 7340216 & 100.00 & 40.7 \\
\hline Micro & 2198687 & 42.2 & 3211197 & 43.7 & 46.1 \\
\hline Pequeña & 899134 & 17.2 & 1287862 & 17.5 & 43.2 \\
\hline Mediana & 364419 & 7.0 & 454721 & 6.2 & 24.8 \\
\hline Grande & 1753568 & 33.6 & 2386436 & 32.5 & 36.1 \\
\hline
\end{tabular}

\%: Participación relativa de cada estrato empresarial en el total del sector servicios.

Fuente: Elaborado con datos de los Censos Económicos 2004 y 2009.

Los servicios aumentaron su ocupación en 2124408 empleos (casi el doble que el comercio, y más de cuatro veces que las manufacturas), de los que 1012510 (47.7\%) corresponden a las EMi. Notablemente, las grandes empresas también contribuyen con un porcentaje alto, $29.8 \%$, equivalente a 632868 nuevos empleos. Los restantes 479030 empleos (22.5\%) fueron generados por las pymes. 


\begin{tabular}{lrcc}
\hline & 2003 & 2008 & Crecimiento 2003-2008 (\%) \\
\hline Servicios & 5.1 & 5.4 & 4.3 \\
Micro & 2.3 & 2.5 & 8.6 \\
Pequeña & 20.5 & 20.0 & -2.4 \\
Mediana & 70.4 & 69.4 & -1.4 \\
Grande & 383.6 & 447.3 & 16.6 \\
\hline
\end{tabular}

Fuente: Elaborado con datos de los Censos Económicos 2004 y 2009 del INEGI.

El cuadro 19 muestra que el promedio de empleados por empresa es también muy bajo: 5.1 en 2003, y 5.4 en 2008; y el promedio en las EMi es de 2.3 y 2.5 respectivamente.

El cuadro 20 contiene el personal ocupado dependiente de la razón social remunerado. Como antes, el porcentaje del personal no remunerado disminuye a medida que el tamaño de las empresas es mayor; otra vez las ME son las que generan menos empleos remunerados.

Cuadro 20. Servicios: personal remunerado

\begin{tabular}{lccccc}
\cline { 2 - 3 } & \multicolumn{2}{c}{ Año 2003 } & \multicolumn{2}{c}{ Año 2008 } \\
\cline { 2 - 3 } \cline { 5 - 6 } Servicios & PODRS remun & \%TD & & PODRS remun & \%TD \\
Micro & 3262497 & 69.8 & & 4211049 & 65.9 \\
Pequeña & 831219 & 39.3 & & 1171052 & 37.8 \\
Mediana & 675677 & 86.2 & & 895801 & 80.9 \\
Grande & 300266 & 97.7 & & 325605 & 93.6 \\
\hline
\end{tabular}

PODRS remun: Personal Ocupado Dependiente de la Razón Social Remunerado \%TD: Porcentaje del personal ocupado dependiente total.

Fuente: Elaborado con datos de los Censos Económicos 2005 y 2009 del INEGI.

Observamos el patrón ya repetido: las EMi tienen un porcentaje muy bajo de personal remunerado, y disminuye de $39.3 \%$ a $37.8 \%$. Los servicios en su conjunto disminuyeron los empleos remunerados de $69.8 \%$ a $65.9 \%$, es decir, $3.7 \%$ menos de empleos remunerados en cinco años; también se observa un retroceso generalizado: todos los estratos disminuyeron sus empleos remunerados.

En el cuadro 21 tenemos los datos para el Valor Agregado Bruto (VAB). El sector presenta una fuerte caída (- $25 \%)$ en el VAB por empleado, principalmente ocasionada por una caída aún más drástica en las EMi, que pasan de producir 91 mil pesos por empleado por año a sólo 52 (- 43.3\%). 
Cuadro 21. Sector servicios: valor agregado bruto (VAB) por estrato empresarial y por empleado, a pesos constantes de 2003

\begin{tabular}{|c|c|c|c|c|c|c|}
\hline & \multicolumn{2}{|c|}{$\begin{array}{l}\text { VAB por estrato } \\
\text { (Millones de pesos) }\end{array}$} & \multirow{2}{*}{$\begin{array}{c}\text { Crecimiento } \\
2003-2008(\%)\end{array}$} & \multicolumn{2}{|c|}{$\begin{array}{l}\text { VAB por empleado } \\
\text { (Miles de pesos) }\end{array}$} & \multirow{2}{*}{$\begin{array}{c}\text { Crecimiento } \\
2003-20081 \%\end{array}$} \\
\hline & 2003 & 2008 & & 2003 & 2008 & \\
\hline Servicios & 905592 & 956309 & 5.6 & 174 & 130 & -25.0 \\
\hline Micro & 200055 & 165682 & -17.2 & 91 & 52 & -43.3 \\
\hline Pequeña & 98977 & 113813 & 15.0 & 110 & 88 & -19.7 \\
\hline Mediana & 50914 & 51896 & 1.9 & 140 & 114 & -18.3 \\
\hline Grande & 555645 & 624918 & 12.5 & 317 & 262 & -17.4 \\
\hline
\end{tabular}

Nota: Pesos de 2008 deflactados con el Índice nacional de precios productor, que se incrementó de 100 en diciembre de 2003, a 136.16 en diciembre de 2008.

<http://dgcnesyp.inegi.org.mx/cgi-win/bdieintsi.exe/Consultar>

Fuente: Elaborado con datos de los Censos Económicos 2005 y 2009.

La disminución en pymes y grandes empresas es menor, pero aún significativa, lo que se debe lógicamente a que el empleo creció más que proporcionalmente. Ya que el valor agregado se compone principalmente de rentas de capital y trabajo, este indicador muestra cómo una recesión tiene dos efectos sobre el producto: por un lado disminuye los niveles de actividad, y por otro disminuye la productividad; es decir, se emplean menos recursos, y los recursos empleados son menos productivos.

En el cuadro 22 tenemos la formación bruta de capital fijo (FBCF). Es notable cómo creció, en términos absolutos, la FBCF en las medianas y grandes empresas (84.7 y 51.7\%), mientras que las micro y las pequeñas presentan un crecimiento negativo $(-4.7 \mathrm{y}-1.0 \%)$. Por cuanto las EMi y las pequeñas empresas combinadas tenían $99 \%$ de las empresas y casi $60 \%$ de los

Cuadro 22. Servicios: formación bruta de capital fijo (FBCF) por estrato y por empleado, a pesos constantes de 2003

\begin{tabular}{|c|c|c|c|c|c|c|}
\hline & \multicolumn{2}{|c|}{$\begin{array}{l}\text { FBCF por estrato } \\
\text { (Millones de pesos) }\end{array}$} & \multirow{2}{*}{$\begin{array}{c}\text { Crecimiento } \\
2003-2008(\%)\end{array}$} & \multicolumn{2}{|c|}{$\begin{array}{l}\text { FBCF por empleado } \\
\text { (Miles de pesos) }\end{array}$} & \multirow{2}{*}{$\begin{array}{c}\text { Crecimiento } \\
2003-2008 \text { (\%) }\end{array}$} \\
\hline & 2003 & 2008 & & 2003 & 2008 & \\
\hline Servicios & 51879 & 71233 & 37.3 & 9.9 & 9.7 & -2.4 \\
\hline Micro & 9107 & 8677 & -4.7 & 4.1 & 2.7 & -34.8 \\
\hline Pequeña & 6525 & 6459 & -1.0 & 7.3 & 5.0 & -30.9 \\
\hline Mediana & 3369 & 6225 & 84.7 & 9.2 & 13.7 & 48.1 \\
\hline Grande & 32877 & 49872 & 51.7 & 18.7 & 20.9 & 11.5 \\
\hline
\end{tabular}

Nota: Pesos de 2008 deflactados con el Índice nacional de precios productor (general y por destino de los bienes finales), que se incrementó de 100 en diciembre de 2003, a 136.16 endiciembre de 2008.

http://dgcnesyp.inegi.org.mx/cgi-win/bdieintsi.exe/Consultar

Fuente: Elaborado con datos de los Censos Económicos 2005 y 2009. 
empleos, el grueso de la economía tuvo un fuerte retroceso, y sólo 1\% de las empresas (las medianas y grandes con $40 \%$ de los empleados) tuvieron una mejoría alta, lo que indica la importancia de incrementar la escala de los negocios. La micro y la pequeña tienen un comportamiento similar.

Por otra parte, la FBCF por empleado confirma el dramático retroceso de las micro y pequeñas empresas, que presentan un cambio de $-34.8 \%$ (de 4.1 mil pesos a 2.7 ) y $-30.9 \%$ (de 7.3 a cinco mil pesos) respectivamente, lo que confirma la pauperización de las micro y pequeñas empresas.

Según el cuadro 23, las remuneraciones por empleado sufrieron una disminución severa (25.2\%), y aunque en este caso las EMi presentan la disminución menos fuerte $(17.8 \%)$, cabe notar que tienen remuneraciones mucho más bajas: para 2008 son casi tres veces menores que las de la pequeña industria, pues descendieron de 1167 a 959 pesos mensuales por empleado.

Cuadro 23. Sector servicios: remuneraciones por estrato empresarial y por empleado, a pesos constantes de 2003

\begin{tabular}{|c|c|c|c|c|c|c|}
\hline & \multicolumn{2}{|c|}{$\begin{array}{l}\text { Remuneraciones por estrato } \\
\text { (Miles de pesos) }\end{array}$} & \multirow{2}{*}{$\begin{array}{l}\text { Crecimiento } \\
2003-2008 \text { (\%) }\end{array}$} & \multicolumn{2}{|c|}{$\begin{array}{l}\text { Remuneraciones } \\
\text { por empleado } \\
\text { (Pesos mensuales) }\end{array}$} & \multirow{2}{*}{$\begin{array}{l}\text { Crecimiento } \\
2003-20081 \%\end{array}$} \\
\hline & 2003 & 2008 & & 2003 & 2008 & \\
\hline Servicios & 284475049 & 299527075 & 5.3 & 4545 & 3401 & -25.2 \\
\hline Micro & 30778474 & 36964067 & 20.1 & 1167 & 959 & -17.8 \\
\hline Pequeña & 37644745 & 42604966 & 13.2 & 3489 & 2757 & -21.0 \\
\hline Mediana & 21514286 & 21192680 & -1.5 & 4920 & 3884 & -21.1 \\
\hline Grande & 194537544 & 198765361 & 2.2 & 9245 & 6941 & -24.9 \\
\hline
\end{tabular}

Nota: Pesos de 2008 deflactados con el Índice nacional de precios productor, que ascendió de 100 en diciembre de 2003, a 136.16 en diciembre de 2008.

<http://dgcnesyp.inegi.org.mx/cgi-win/bdieintsi.exe/Consultar>

Fuente: Elaborado con datos de los Censos Económicos 2005 y 2009 del INEGI.

\section{Sector comercio}

En esta sección llevamos a cabo, para el comercio, el mismo análisis que realizamos antes para manufacturas y servicios, pero prescindimos de los cuadros de datos, para evitar cierta repetitividad. En general, los resultados son similares, con algunas particularidades que detallamos en lo que sigue.

Para 2008, el comercio disponía de casi la mitad de las empresas del país, lo que indica un retroceso de $2.7 \%$ que tiene como contraparte un aumento en la participación de los servicios, como ya vimos. La participación en el 
empleo se mantuvo casi constante, mientras que en la producción bruta retrocedió en $3.4 \%$.

La estructura empresarial no tuvo variaciones significativas, pues en todos los casos los cambios observados en la participación de cada estrato son menores a 1\%. El número total de empresas creció a 277963, de las cuales 269934 fueron EMi, con lo cual éstas incrementaron su participación de $97 \%$ a $97.1 \%$; es decir, las pequeñas, medianas y grandes empresas combinadas apenas alcanzan el $2.9 \%$.

Como ya dijimos, en términos del desarrollo económico esto constituye un indicador negativo, pues cuanto mayor y más persistente es el número de EMi, menor integración y desarrollo se observa en las pymes, las cuales podrían constituir la columna vertebral de un desarrollo económico pujante.

A diferencia de la industria manufacturera, en el comercio las EMi participan con la mayor parte del empleo del sector: $62.1 \%$ en 2003 , y $65.1 \%$ en 2008; esto es, en cinco años hubo un aumento de 1137392 empleos, de los que 892439 (78.5\%) fueron generados por las EMi. Esto es nuevamente un proceso de pauperización de la economía, en el sentido de que la mayor participación y el mayor crecimiento del empleo, se dan en el estrato más empobrecido y con menor potencial: el de las EMi.

La estructura del número promedio de empleados por estrato no tuvo cambios significativos; pero la media en las EMi es aún más baja que en la manufactura: las EMi tienen aquí 7.6 veces menos empleados promedio que la pequeña empresa en 2008.

Con respecto al personal ocupado dependiente de la razón social remunerado, otra vez el porcentaje del personal no remunerado disminuye a medida que el tamaño de las empresas es mayor. Igual que en las manufacturas, las EMi generan menos empleos remunerados. Pero su situación es mucho más precaria. El comercio disminuyó la participación de los empleos remunerados de $52.7 \%$ a $45.1 \%$ (la disminución en las manufacturas fue de $87.8 \%$ a $82.1 \%$ ), es decir, $7.6 \%$ menos empleos remunerados en cinco años, en un retroceso generalizado.

Una importante característica de la estructura empresarial del sector comercio, es que la diferencia entre el porcentaje de empleos remunerados en la EMi y demás estratos es aún más abismal que en las manufacturas; las EMi sólo remuneraban a $28.3 \%$ de sus empleados en 2003, y disminuyeron este porcentaje a $26.4 \%$ en 2008 ; para la pequeña empresa, los porcentajes fueron 95.3 y 91.6, respectivamente; hay otra vez un comportamiento similar EMi-pequeña vs. mediana-grande.

Observamos un doble efecto negativo: por un lado, las EMi tienen una tasa extraordinariamente baja de empleos remunerados; por el otro, dismi- 
nuyen aún más esa tasa de 2003 a 2008. El hecho de que las pymes también disminuyeron la tasa de empleos remunerados, constituye una indicación más del empobrecimiento estructural sufrido por la economía en ese periodo. Es sintomático que más de la mitad de los empleos del sector comercio (54.9\%), pasaron a ser no remunerados en 2008.

En lo que se refiere al valor agregado bruto (VAB), hay una disminución generalizada (-20.5\%) para el sector en su conjunto. La mayor disminución se observa nuevamente en la EMi (-29\%), y luego en la pequeña (-21\%). El VAB por empleado disminuye aún más drásticamente (-35.2\%): el sector pasa de generar 116 mil pesos por empleado en 2003, a 75 mil en 2008 . Y otra vez, la reducción más severa está en las EMi: de 66.8 a 36.5 (-45.3\%). Aunque sería necesario realizar una investigación sobre las causas y mecanismos específicos que operan esta disminución, el hecho es que precisamente el producto per cápita (en este caso aproximado por el vAB por empleado) es el principal indicador de la competitividad de una economía, y lo que posibilita la mejora de salarios y de niveles de vida. El deterioro de este indicador causa el deterioro salarial y, en general, el deterioro del bienestar; en efecto, no es difícil mostrar que el poder adquisitivo en México ha mantenido una sostenida disminución durante el periodo 2003-2008, e incluso desde antes.

Con respecto a la Formación Bruta de Capital Fijo (FBCF), una característica importante es que la estructura por empleado es básicamente la misma en 2003 y en 2008. La EMi presenta la más baja, de 1.7 a 1.8, y por alguna razón se presenta un extraordinario crecimiento en las grandes empresas: de 8.2 a 17.4 mil pesos por empleado.

Por otra parte, las remuneraciones absolutas disminuyeron en $15.6 \%$, como resultado de una caída generalizada; y por empleado disminuyen más fuertemente (31.3\%), como producto de una disminución similar en los estratos, excepto por las grandes, que decrecieron en $36 \%$.

El punto es que las EMi son las que tienen siempre las menores remuneraciones por empleado: 674 pesos mensuales en el 2008, 4.4 veces menos que las del estrato siguiente, que son las pequeñas empresas con 2988 pesos mensuales.

\section{Algunas conclusiones y reflexiones finales}

Las principales conclusiones de esta investigación se derivan del análisis detallado de la estructura empresarial mexicana, y de sus características y desempeño, durante los años 2003 y 2008, según datos de los Censos Económicos 2004 y 2009. 
Se podría argumentar que la validez de las conclusiones es cuestionable, en la medida en que 2008 no fue un año normal, o típico, de la economía mexicana (para la que no es fácil encontrar periodos de desarrollo "normal" o "típico"; al contrario, su evolución, desde la "década perdida" de los ochenta, parece estar caracterizada por altibajos diversos, que han contribuido a la "pérdida" de las siguientes décadas), sino el resultado de una crisis exógena, la llamada crisis financiera de 2008. Sin descartar la importancia del impacto de esa crisis sobre el desempeño del país, destacamos los siguientes puntos.

El análisis comparativo fundamenta sólo una parte de los resultados; por otra parte, esa crisis comenzó a afectar la economía mexicana hacia el 2008, por lo que es de suponer que no determinó significativamente su desempeño durante ese año, y que su influencia fue más bien marginal, pues el impacto más fuerte se produjo a lo largo de 2009.

Aun suponiendo que la crisis fuese determinante en el desempeño de 2008, no es razonable suponer que en ese mismo año causara cambios significativos en la estructura industrial del país (o que los evitara); por lo tanto, la crisis y su impacto son irrelevantes para nuestro análisis.

Además, el análisis comparativo se hace aquí para dos estados de la economía distanciados por un periodo de cinco años; si un indicador muestra que un aspecto de la economía es mejor o peor en 2008 que en 2003, ese aspecto es mejor o peor independientemente de la causa que lo haya originado. El hecho es que el estado de la economía en un periodo dado es el que reflejan los datos, y su subsecuente evolución tendrá lugar a partir de ahí.

Pasando ahora a las conclusiones, los resultados indican que la estructura empresarial no experimentó cambios cualitativos de importancia; es decir: la participación relativa de los cuatro estratos empresariales definidos, se mantuvo relativamente constante y, por consiguiente, las variaciones cuantitativas fueron no significativas. Por otra parte, en los indicadores de desempeño se observa una drástica caída, particularmente en aquellos relacionados con el potencial desarrollo de la economía: producción de valor agregado por empleado (productividad) y formación bruta de capital fijo también por empleado (inversión física). Es de particular interés notar que las remuneraciones reales pagadas por los sectores productivos en 2008, observaron una disminución generalizada en torno a $25 \%$ en relación con las pagadas en 2003; es decir, los trabajadores ganaban una cuarta parte menos.

Con respecto a las pymes, el análisis de la información censal indica que, en general, no incrementaron su participación en la economía; por el contrario, la mayor parte de las veces su participación disminuyó; esas disminuciones generalmente se correspondieron con un incremento en la 
participación del estrato de las EMi, lo cual, habida cuenta las pésimas condiciones en que éstas se encuentran, significa que el estado de la economía mexicana, en 2008, presentaba un retroceso generalizado con respecto a su situación en 2003. Así pues, los resultados de nuestro análisis avalan la hipótesis de una tercera década perdida; más aún, los datos recogidos por los censos muestran que la economía se dirige hacia una cuarta década perdida, en términos de su desarrollo.

Como apuntamos, la razón capital-trabajo es una de las claves fundamentales para el desarrollo de una economía, pues consistentemente se observa que los países que se desarrollan, y los desarrollados, tienen una alta y creciente razón capital-trabajo. Por ello es relevante considerar la FBCF en las pymes en relación con los demás estratos, y en particular con las EMi: en los sectores manufacturas y comercio, la FBCF por empleado en las pequeñas empresas es aproximadamente cuatro veces mayor que en las EMi, y casi el doble en el sector servicios. Esto demuestra que a medida que se hace mayor la participación del estrato micro en la economía mexicana, tanto más se pauperizan ésta y sus posibilidades de desarrollo.

El hecho de que ya en 2003 la estructura empresarial se encontraba en un estado deplorable, aunado al retroceso que sufre en 2008, conduce al menos a dos conclusiones: a) la situación de la estructura empresarial y sus cambios de 2003 a 2008, corroboran que 2001-2010 fue una década perdida; y b) tal estructura implica que, en lo que concierne al crecimiento económico, la actual será también una década perdida, por la simple razón de que es físicamente imposible que tal estructura dé lugar a un desarrollo económico sostenido y significativo; por otra parte, una estructura empresarial que sí pudiera lograrlo es imposible de construir en un sexenio. Como cita Sagasti (2011: 16): “Jorge Sábato, el ilustre pionero de la política científica y tecnológica en la región solía decir que 'toma quince años crear una institución de investigación de nivel mundial, pero solo dos años destruirla' ". Siendo realistas, lo que queda por hacer es procurar que sea una década "ganada" en términos sociopolíticos, de tal modo que se puedan sentar las bases para un posterior crecimiento sostenible y duradero.

Las causas directas o inmediatas - en contraposición a las causas mediatas o últimas - son relativamente fáciles de identificar y analizar, de modo que puede ser relativamente sencillo elaborar recomendaciones de política dirigidas a impulsar el desarrollo. En nuestro caso, una vez identificadas las problemáticas de la baja productividad y de la baja inversión en capital físico, la evidente recomendación de política sería la de implementar programas que incrementen la productividad y que estimulen la 
FBCF. Pero al llegar al diseño e implementación de tales programas, toparíamos con el problema de fondo; en primer lugar: ¿por qué disminuyó la inversión en capital físico?

El estancamiento económico es un fenómeno complejo que obedece a diversos factores igualmente complejos; factores que, en contraposición a las causas inmediatas o directas, podemos llamar causas últimas, cuya complejidad dificulta su análisis y sistematización, pues se trata de cuestiones inherentes a la estructura propia de la sociedad y a las pulsiones internas que motivan su evolución; y tanto la estructura social como su dinámica están a su vez configuradas por otra serie de factores complejos: la historia, las particularidades culturales, la geografía, las instituciones y demás.

Según Moreno-Brid y Ros-Bosch (2011), la baja tasa de inversión obedece a cuatro factores: disminución de inversión pública, apreciación del tipo de cambio real (para controlar la inflación pero que disminuye la rentabilidad de las ramas productoras de comerciables), desmantelamiento de incentivos a la inversión (política industrial), y un restringido acceso al financiamiento. Pero otra vez tenemos que preguntar: ¿por qué se dio esto, qué lo causó en última instancia?

A partir de lo anterior, estos autores concluyen, obviamente, que para lograr un crecimiento elevado y sostenido es necesario recuperar altas tasas de inversión pública en infraestructura, una política monetaria que impida apreciaciones significativas y persistentes, fortalecer el sistema financiero para otorgar créditos al sector productivo, y una política moderna de desarrollo sectorial que impulse la innovación, la inversión en nuevas actividades, etc. La pregunta es, desde luego, ¿cómo?

En México, el déficit democrático, causado por diversos factores, ha ocasionado la persistencia en el poder de cúpulas poco o nada comprometidas con el desarrollo del país, por lo cual consideramos, según lo argumentado en la sección 2, que el avance político, y en particular el desarrollo de una democracia efectiva, constituye un requisito indispensable para iniciar un desarrollo económico y social sólido y duradero.

\section{Referencias}

Bergoeing, R. et al., 2001, “A Decade Lost and Found: Mexico and Chile in the 1980s", NBER, Working Paper 8520.

Easterly, W., 2001, “The Lost Decades: Developing Countries' Stagnation in Spite of Policy Reform 1980-1998", Journal of Economic Growth, vol. 6, núm. 2. 
INEGI, 2011, Censos Económicos 2009.

INEGI, 2010ª Metodología de los Censos Económicos 2009.

INEGI, 2010b, Resumen de los resultados de los Censos Económicos 2009.

INEGI, 2008, Información sobre micronegocios sin local, Encuesta Nacional de Micronegocios, Enamin 2008.

INEGI, 2006, Micro, pequeña, mediana y gran empresa. Estratificación de los establecimientos.

INEGI, 2005, Resumen general Censos Económicos 2004.

INEGI, 2005, Metodología de los Censos Económicos 2004.

INEGI, 2005, Industrias manufactureras Censos Económicos 2004.

INEGI, 2005, Comercio al por mayor Censos Económicos 2004.

INEGI, 2005, Comercio al por menor Censos Económicos 2004.

INEGI, 2005, Censos Económicos 2004.

Moreno-Brid, J.C. y J. Ros-Bosch, 2011, “El desarrollo a largo plazo de la economía mexicana: 1810-2008", Institucionalidad y desarrollo económico en América Latina, CEPAL.

Sagasti, F., 2011, Ciencia, tecnología, innovación. Políticas para América Latina, Lima, Perú, FCE.

Santiso, C., 2003, "Another Lost Decade? The Future of Reform in Latin America", Public Administration and Development, 23.

Recibido el 13 de noviembre de 2011 Aceptado el 4 de septiembre de 2012 